\title{
Systematic literature review of hospital medication administration errors in children
}

This article was published in the following Dove Press journal:

Integrated Pharmacy Research and Practice

5 November 2015

Number of times this article has been viewed

\author{
Ahmed Ameer' \\ Soraya Dhillon' \\ Mark J Peters ${ }^{2}$ \\ Maisoon Ghaleb' \\ 'Department of Pharmacy, School of \\ Life and Medical Sciences, University \\ of Hertfordshire, Hatfield, UK; \\ 2Paediatric Intensive Care Unit, Great \\ Ormond Street Hospital, London, UK
}

Correspondence: Ahmed Ameer Department of Pharmacy, University of Hertfordshire, College Lane, Hatfield, Hertfordshire ALI0 9AB, UK

Tel +44 I707 284248

$\mathrm{Fax}+441707284506$

Email ahmed.ameer@nhs.net
Objective: Medication administration is the last step in the medication process. It can act as a safety net to prevent unintended harm to patients if detected. However, medication administration errors (MAEs) during this process have been documented and thought to be preventable. In pediatric medicine, doses are usually administered based on the child's weight or body surface area. This in turn increases the risk of drug miscalculations and therefore MAEs. The aim of this review is to report MAEs occurring in pediatric inpatients.

Methods: Twelve bibliographic databases were searched for studies published between January 2000 and February 2015 using "medication administration errors", "hospital", and "children" related terminologies. Handsearching of relevant publications was also carried out. A second reviewer screened articles for eligibility and quality in accordance with the inclusion/exclusion criteria.

Key findings: A total of 44 studies were systematically reviewed. MAEs were generally defined as a deviation of dose given from that prescribed; this included omitted doses and administration at the wrong time. Hospital MAEs in children accounted for a mean of $50 \%$ of all reported medication error reports $(n=12,588)$. It was also identified in a mean of $29 \%$ of doses observed $(\mathrm{n}=8,894)$. The most prevalent type of MAEs related to preparation, infusion rate, dose, and time. This review has identified five types of interventions to reduce hospital MAEs in children: barcode medicine administration, electronic prescribing, education, use of smart pumps, and standard concentration.

Conclusion: This review has identified a wide variation in the prevalence of hospital MAEs in children. This is attributed to the definition and method used to investigate MAEs. The review also illustrated the complexity and multifaceted nature of MAEs. Therefore, there is a need to develop a set of safety measures to tackle these errors in pediatric practice.

Keywords: medication administration errors, children's hospital, pediatric, nature, incidence, intervention

\section{Introduction}

Children are a large, vulnerable group, who undergo rapid growth and development. They face many challenges in this process of development that may require health care professionals' advice and a hospital stays. These challenges are most likely to be treated or managed by the use of medications, which can potentially be subject to a medication error (ME).

MEs have been defined as "errors in drug ordering, transcribing, dispensing, administering, or monitoring" ${ }^{1}$ These errors are mostly preventable and constitute the most common type of adverse drug events. Kohn et $\mathrm{al}^{2}$ described an adverse drug event as an injury resulting from medical intervention related to a drug, which can be attributable 
to preventable and non-preventable causes. Administration of medicine is the last process of the medication cycle for errors to be detected prior to patients receiving their treatment.

Errors occurring in the administration process could be attributed to the fact that children receive small doses. These doses are usually prepared from pharmaceutical formulations expressed in strengths that are not child-friendly. ${ }^{3,4}$ Moreover, in pediatric medicine, doses are usually calculated based on the child's weight or body surface area. ${ }^{5}$ Therefore, calculation errors are possible in both the prescribing and administration stages that could result in a tenfold or more overdose. ${ }^{6}$

Furthermore, most medications are given to children on an unlicensed and/or off-label basis. ${ }^{7}$ Unlicensed medicines are used without a children-specific use license from the medicine regulatory authorities. Whereas off-label use of medicine is referring to using a medicine for an indication that falls outside the licensed indications set by the medicine regulatory authorities. There is limited evidence suggesting that unlicensed and off-label use of medications is associated with the high frequency of MEs. ${ }^{7}$ This could be due to limited prescribing information in children for these uses.

There are different methods used to investigate medication administration errors (MAEs) in practice. ${ }^{8}$ The different methodological approaches could cause confusion and complexity in understanding the true nature of the problem since various denominators are used such as the number of doses observed, number of incident reports, and number of opportunities for errors. ${ }^{6,8,9}$

One of the earliest reviews in this area was by Ghaleb et $\mathrm{al}^{6}$ that identified incidences of MAEs between 0.6 and 27 per 100 administered doses in children's hospitals. This review focused on definitions, incidence, and prevalence of MEs relating to prescribing and administration only. The review by Ghaleb et $\mathrm{al}^{6}$ explored the need to develop validated definitions of prescribing and administration errors. This will help in understanding the true scale of the problem and to test interventions for reducing these errors. Another systematic review by Miller et $\mathrm{al}^{10}$ found an MAE rate between 72 and 75 in every 100 reported MEs. This review recommended unifying numerators and denominators when collecting data and standardization of definitions. Rinke et $\mathrm{al}^{11}$ reached a similar conclusion with their review on interventions that were used to reduce MEs. It is of interest to note that hospital MAEs in children are more prevalent than adults. A systematic review found that hospital MAEs in children occur between 17.4 and 33.8 per 100 opportunities for error. ${ }^{9}$ On the other hand, prevalence of MAEs in adults is between 4.7 and 27.8 per 100 opportunities for error. ${ }^{9}$
A systematic literature review ${ }^{12}$ found that MAEs are mainly caused by human related factors and other causes such as health care professionals' knowledge-based mishaps, workload, distractions/interruptions, and staffing levels.

There have been attempts to reduce MAEs by lowering the number of interruptions. However, as demonstrated by a systematic review ${ }^{13}$ of ten studies, weak supporting evidence is found for this claim. Therefore, to effectively reduce MAEs, interventions need to address the multifaceted nature of MAEs.

Other systematic reviews in this field did not explore thoroughly all the evidence relating to hospital MAEs in children. In particular, there is a need to review the interventions evaluated to reduce MAEs in children's hospitals. Therefore, this systematic review aims to investigate all studies of hospital MAEs in children. The review objectives are to: 1) explore definitions used to identify hospital MAEs in children, 2) report the prevalence of hospital MAEs in children, 3) identify the nature and severity of these errors, and 4) identify the interventions used to reduce hospital MAEs in children.

\section{Methods}

\section{Data sources and search terms}

Studies were searched in March 2015 from 12 databases, which include PubMed, Science Direct, Web of Knowledge, British Nursing Index, Scopus, Global Health, EMBASE, NeLM, CINAHL, International Pharmaceutical Abstracts, PsycInfo, and PsycExtra. The following search terms were used: ("Medication Error" OR "Medication mistake" OR "Drug error" OR "Drug mistake" OR "Drug mishap" OR "Adverse drug event" OR "Near Miss" OR "Death") AND ("Administration Error" OR "Medication administration error" OR "Drug administration mistake" OR "Drug administration" OR "preparation error") AND ("Hospital" OR "Secondary care" OR "Tertiary centre") AND ("Paediatric" OR "pediatric" OR "Child" OR "Infant" OR "Adolescent" OR "Toddler" OR "Neonate" OR "Newborn" OR "Under 16" OR "Teen" OR "Teenager" OR "Baby"). Also, handsearching of relevant publications from recent systematic reviews to identify all possible studies was carried out.

\section{Selection criteria}

The following inclusion and exclusion criteria were used to select studies:

1. Publication date between January 1,2000 and February 28,2015 . This is to avoid repetitiveness of findings since earlier studies have been reviewed by other researchers and build on existing reviewed evidence. 
2. Presented in English language. Studies that are not available in English require a different set of search terms. These search terms will need to be in different languages to cover all possible languages. Also to avoid bias, nonEnglish literature databases will need to be searched. Moreover, professional interpreters with research background would be required to search, retrieve the studies, and extract data for these reviews.

3. MAEs in hospital settings in children aged between 0 and 18 years old. This is to reflect the population of interest for this research. This will also ensure that the data collected relate only to the children's hospital setting.

4. MAEs related to the use of total parenteral nutrition were not selected. This is to reduce heterogeneity of the findings.

All articles that are not peer reviewed such as opinions, letters, comments, editorials, and reviews studies were excluded. However, they were used to handsearch for additional studies from their bibliographies to ensure that all potential studies were captured. Studies that did not report child data were also excluded.

\section{Quality assessment and extraction process}

The researchers retrieved studies for review from the above databases using the search strategy. A rigorous review to assess suitability against the review criteria was carried out. An experienced researcher from the Department of Pharmacy at University of Hertfordshire reviewed all articles identified for quality assurance and validity purposes. A high level of agreement was established between the two reviewers and the studies that were in disagreement $(n=4)$ were resolved through a discussion and by referring back to the criteria set. There was no need for a third opinion.

The Preferred Reporting Items for Systematic Reviews and Meta-Analyse ${ }^{14}$ standards were used to extract data and assess the quality of the studies. Citations were imported into a reference manager EndNote X7 (Thomson Reuters, New York, NY, USA). Microsoft Excel 2010 (Microsoft Corporation, Redmond, WA, USA) was used to store data extracted from the selected articles. The following data were recorded: authors, year of publication, country of origin, study type, setting, duration, MAE definitions used, method of identifying MAEs, key findings, and recommendations.

\section{Data analysis}

Data extracted from each study were aggregated into three categories: 1) studies that reported specific definition for hospital MAEs in children, 2) investigational studies that found the nature of hospital MAEs in children without testing an intervention, and 3) studies that evaluated the effectiveness of an intervention. Basic descriptive analysis of data was conducted for studies that used the same numerator and denominator of MAEs.

\section{Results \\ Database search results}

The search strategy found 3,466 articles. As illustrated in Figure 1, 3,422 articles were eliminated in compliance with the inclusion/exclusion criteria. This yielded 44 studies that were found to be eligible for the purpose of this review. These studies were carried out in the following countries: USA $(n=16) ;{ }^{1,15-29}$ UK $(n=8) ;, 30-36$ Canada $(n=4) ; 3^{37-40}$ France $(\mathrm{n}=2) ;{ }^{41,42}$ Germany $(\mathrm{n}=2) ;{ }^{43,44}$ Malaysia $(\mathrm{n}=2) ;{ }^{45,46}$ Argentina $(n=1) ;{ }^{47}$ Australia $(n=1) ;{ }^{48}$ Ethiopia $(n=1) ;{ }^{49}$ Japan $(n=1) ;{ }^{50}$ the Netherlands $(n=1) ;{ }^{51}$ Saudi Arabia $(n=1) ;{ }^{52}$ Spain $(n=1) ;{ }^{53}$ Switzerland $(\mathrm{n}=1) ;{ }^{54}$ Turkey $(\mathrm{n}=1) ;{ }^{55}$ and multicenter $(\mathrm{n}=1) .{ }^{56}$

Table 1 represents a summary of all the 44 studies that were reviewed. It contains the core information for each study and an overview of the key findings. As can be seen, the studies were themed into three groups: studies that used

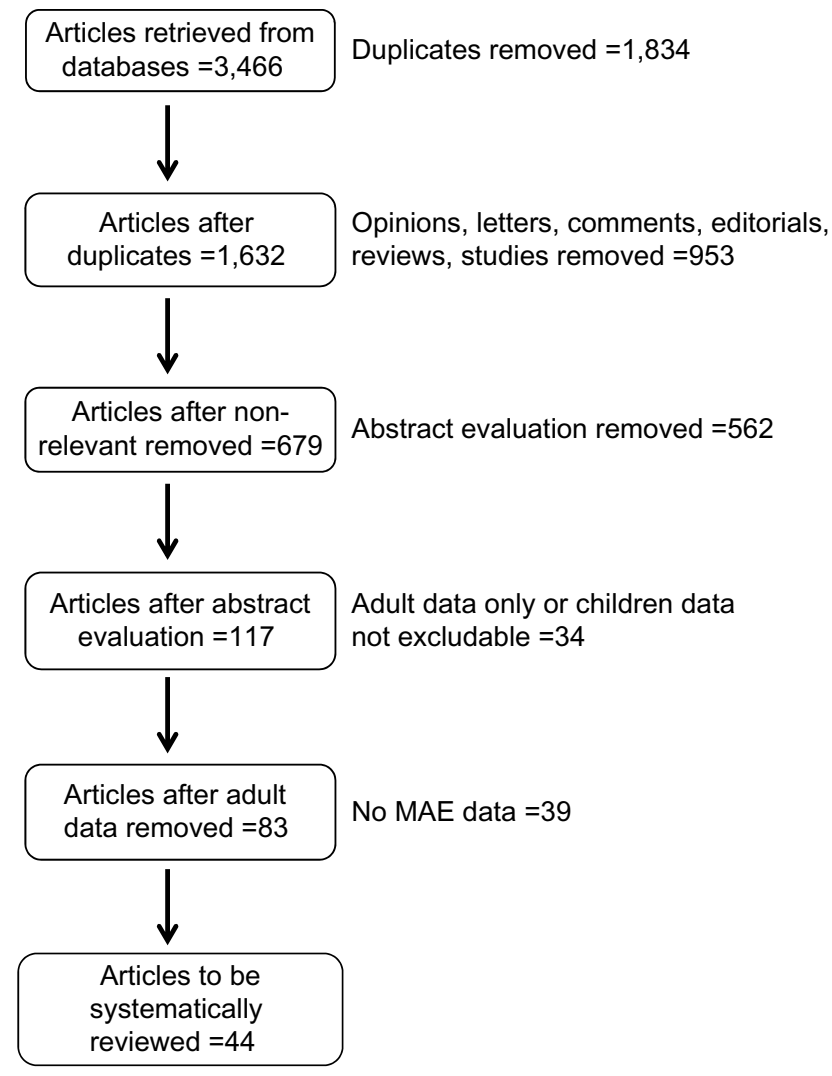

Figure I Flow diagram showing article selection for the systematic literature review. Abbreviation: MAE, medication administration error. 



क व 20
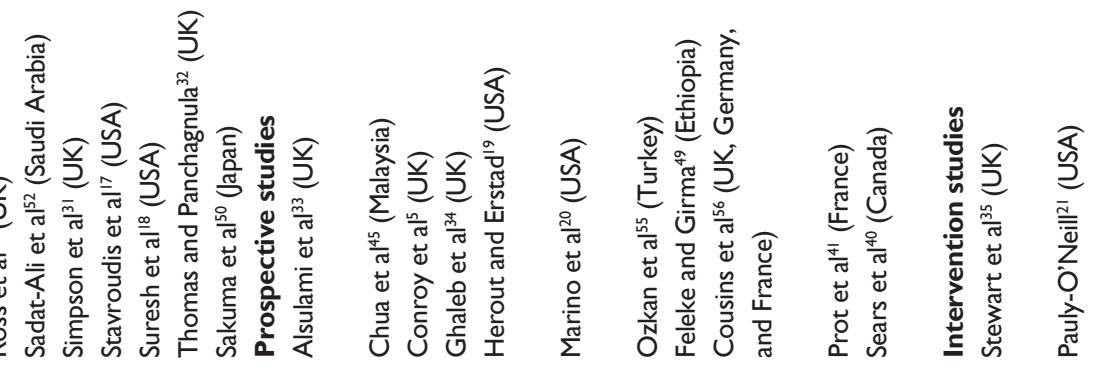


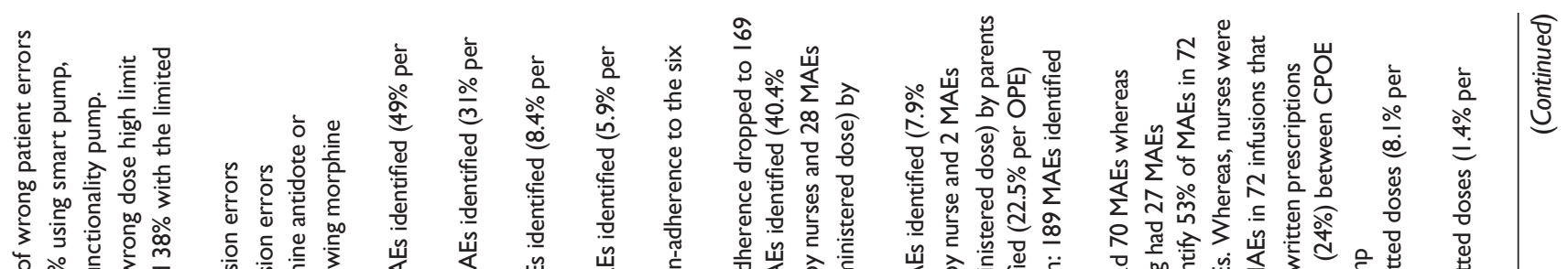

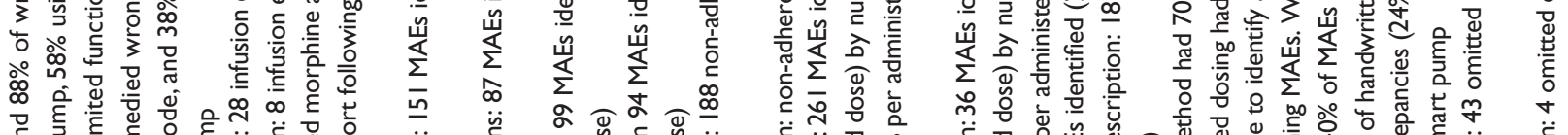

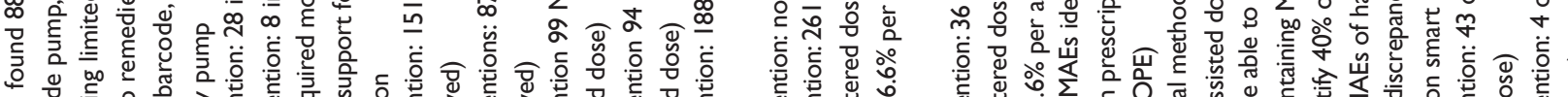

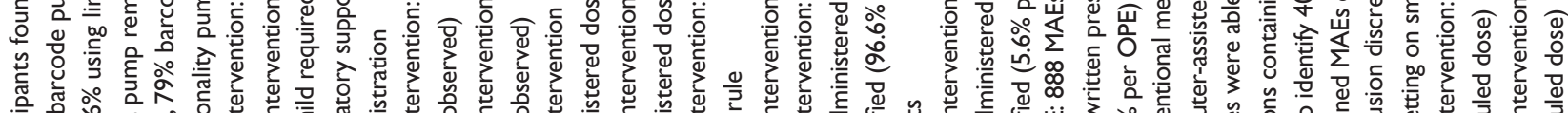

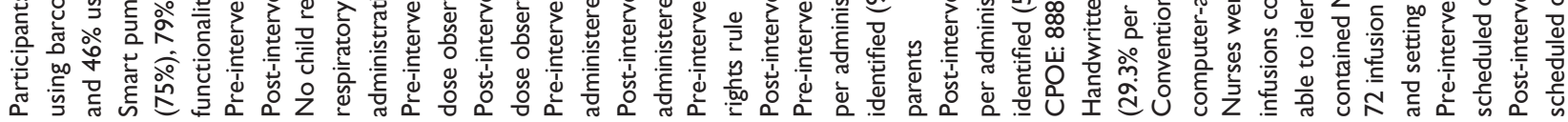

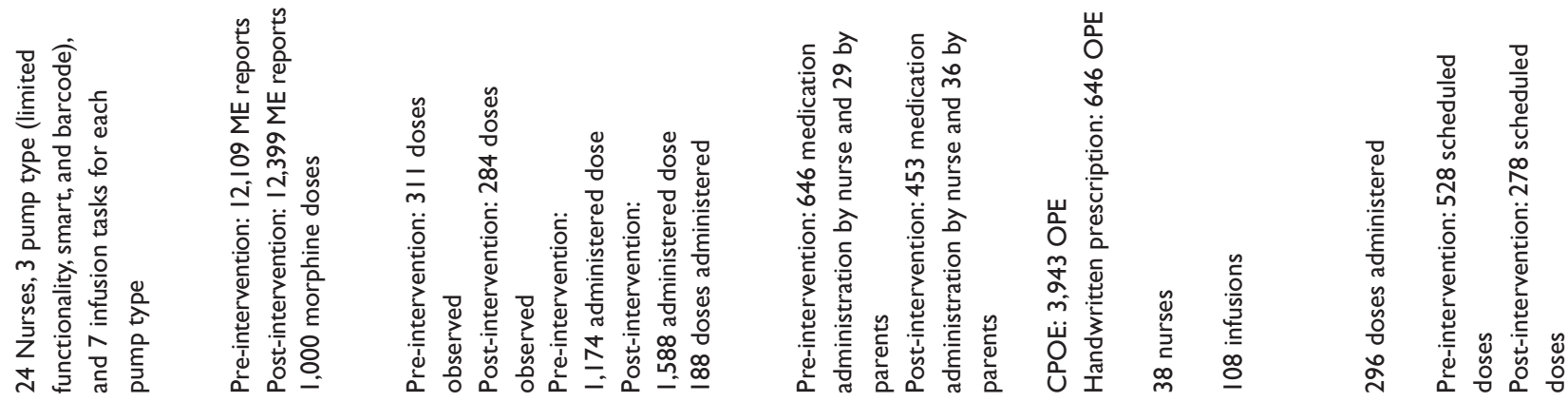

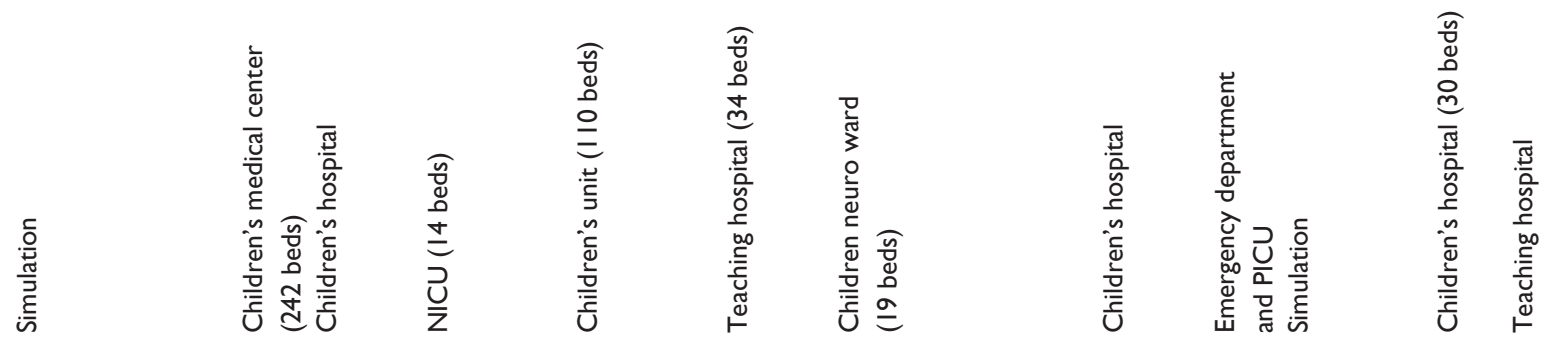



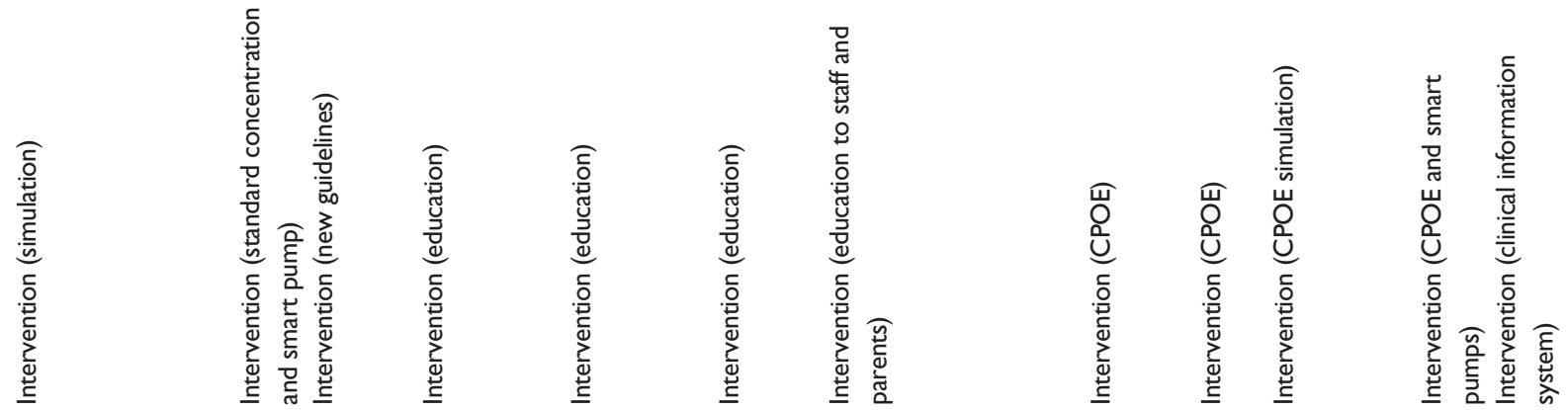

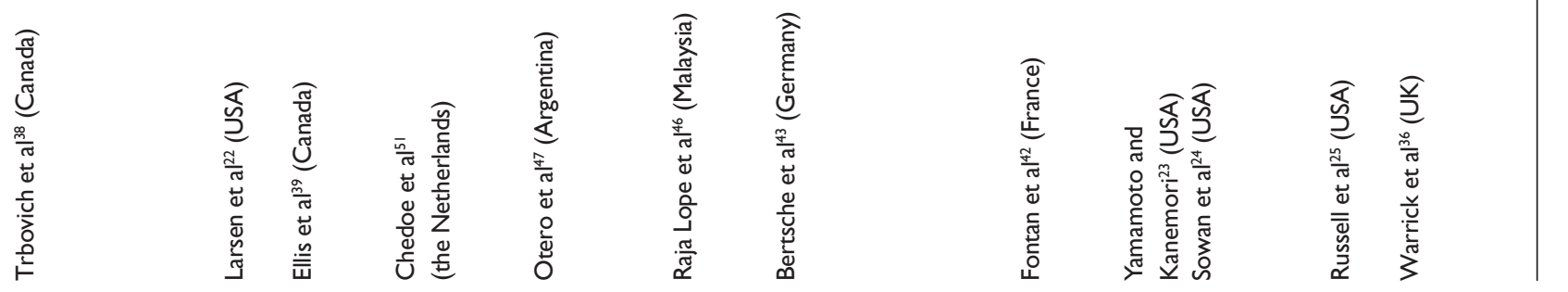




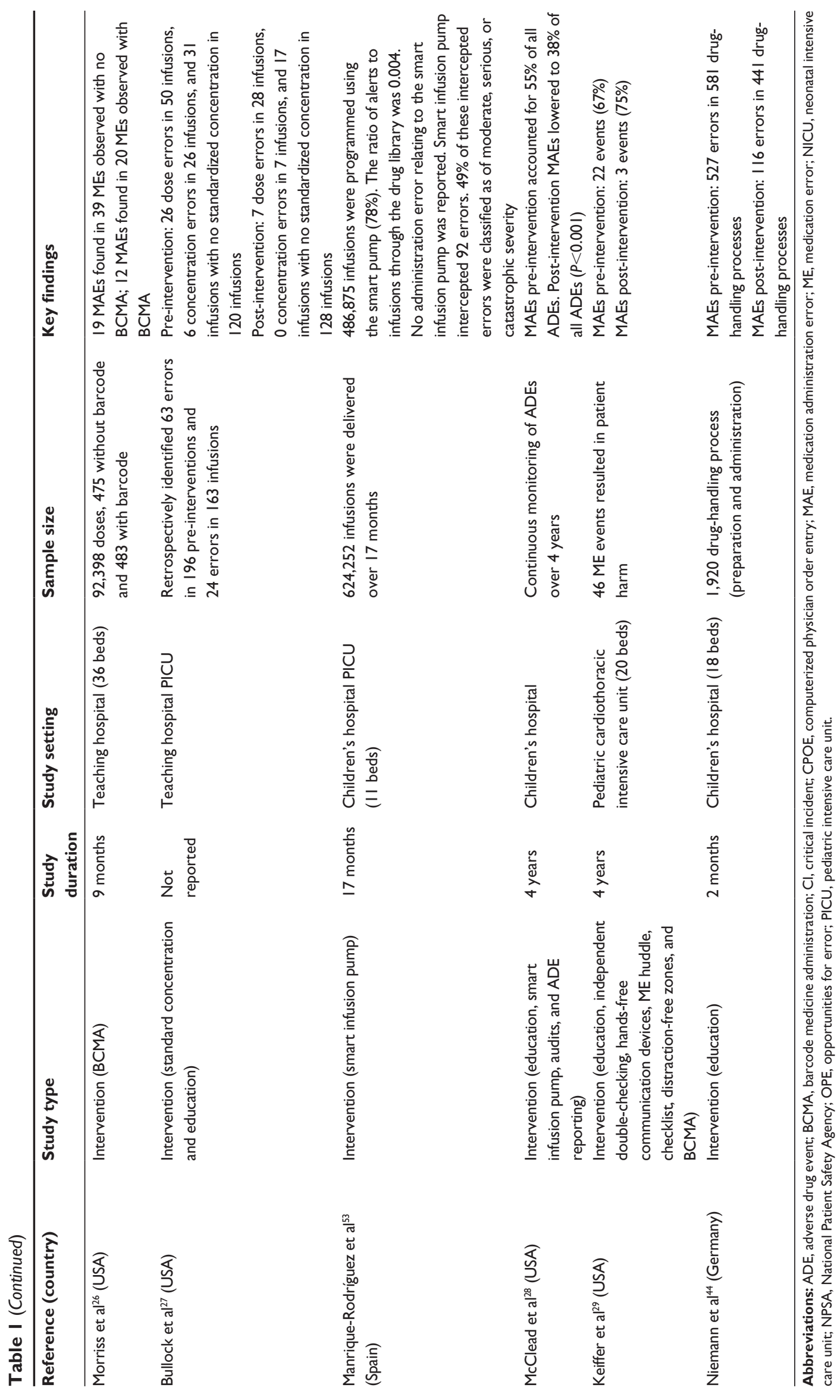


a retrospective approach to investigate MAEs, prospective observational studies, and studies that investigated the effect and impact of an intervention.

\section{Definitions of hospital MAEs in children}

There were eight studies that reported a specific definition for hospital MAEs in children. ${ }^{19,34,41,42,45,46,49,56}$ Table 2 illustrates the key components of the definitions found. As can be seen, MAEs can generally be defined as a variation of the dose given from that originally prescribed. The remaining studies did not report a specific MAE definition. However, they utilized a broad ME definition such as the one by Kaushal et $\mathrm{al}^{1}$ which describes MEs as "errors in drug ordering, transcribing, dispensing, administering, or monitoring".

\section{Prevalence of hospital MAEs in children}

Studies that investigated the prevalence of hospital MAEs in children have used two methodological approaches. The first was a retrospective method that included critical incident review, ${ }^{18,32}$ analysis of ME specific incident reports, ${ }^{15-17,30,31,37,48,50,52,54}$ and review of medication charts. ${ }^{1,20}$ The second methodological approach was using a prospective method. This was in the form of either undisguised observation of the medication administration process, ${ }^{19,33,34,41,45,55-57}$ or disguised observations. ${ }^{49,56} \mathrm{~A}$ further study ${ }^{40}$ was identified that prospectively collected self-reported MAEs spontaneously.

\section{Retrospective methods}

As can be seen in Table 1, retrospective studies are a commonly used method to investigate MAEs. This approach was utilized by 14 studies to investigate hospital MAEs in children. ${ }^{1,15,16,18,20,30-32,37,48,50,52,54,58}$ It involves the review of records kept by health care professionals that are in the form of critical incident reports, ME reports, or medication charts.

Critical incident reports were included MAEs as part of all other patient safety incidents. Two studies ${ }^{18,32}$ using this approach found 362 MAEs in 13,314 critical incidents relating to children in hospital care. This is the equivalent of a prevalence rate between 12 and 15 MAEs in every 100 critical incident reports.

Whereas, ME reports relate specifically to incidents of medication use. This includes prescribing, dispensing, and administration errors. This approach was used in ten studies. ${ }^{15,16,30,31,37,48,50,52,54,58}$ Despite the heterogeneity of data, there were 12,588 ME reports in children's hospitals and MAEs accounted for $50 \%$ of the reports $(n=6,247)$. This yields a prevalence of 29 to 76 hospital MAEs in every $100 \mathrm{ME}$ reports in children.

Another method found was to identify MAEs from medication charts. Screening medication charts for MEs is carried out first. This is then represented in a breakdown of all ME types including MAEs. Studies that used this method found a prevalence of between 2 and 13 MAEs in every 100 MEs identified. ${ }^{1,20}$ There was no study that has reviewed medical records to identify MAEs.

\section{Prospective methods}

The second approach that was found to investigate hospital MAEs in children is the use of prospective observation of medication administration practice. This is conducted in disguised or undisguised manner. The prevalence of MAEs

Table 2 Key definition components used to investigate medication administration errors (MAEs) in children's hospitals

\begin{tabular}{|c|c|c|c|c|c|}
\hline \multirow[t]{3}{*}{ Study } & \multicolumn{5}{|c|}{ Definition components } \\
\hline & \multicolumn{3}{|c|}{ MAE is variation of dose given from } & \multirow{2}{*}{$\begin{array}{l}\text { Preparation } \\
\text { errors }\end{array}$} & \multirow[t]{2}{*}{ Other } \\
\hline & Prescription & $\begin{array}{l}\text { Hospital } \\
\text { procedures }\end{array}$ & $\begin{array}{l}\text { Manufacture } \\
\text { procedures }\end{array}$ & & \\
\hline Chua et al ${ }^{45}$ & $\checkmark$ & $\checkmark$ & & & \\
\hline Cousins et $\mathrm{al}^{56}$ & $\checkmark$ & $\checkmark$ & $\checkmark$ & $\checkmark$ & \\
\hline Feleke and Girma ${ }^{49}$ & & & & & $\begin{array}{l}\text { "Occurs while administering a } \\
\text { medication to a patient" }\end{array}$ \\
\hline Fontan et $\mathrm{al}^{42}$ & $\checkmark$ & & & & \\
\hline Ghaleb et $\mathrm{al}^{134}$ & $\checkmark$ & $\checkmark$ & & $\checkmark$ & \\
\hline Herout and Erstad ${ }^{19}$ & $\checkmark$ & & & & $\begin{array}{l}\text { Includes omission; dosing errors } \\
\text { for weight-based infusion were } \\
\text { defined as a } 5 \% \text { difference }\end{array}$ \\
\hline Prot et $\mathrm{al}^{41}$ & $\checkmark$ & & & & \\
\hline Raja Lope et al ${ }^{46}$ & & & & & $\begin{array}{l}\text { Process of "commission and } \\
\text { omission" by nurse }\end{array}$ \\
\hline
\end{tabular}


is dependent on the denominator used. The studies that measured MAEs against the number of doses observed have found 9 to 90 MAEs in every 100 doses observed. $5,19,33,41,45,49,55,56$ Despite heterogeneity, cumulatively this represents a total of 2,537 children's hospital MAEs (29\%) identified in 8,894 doses observed.

As presented in Table 1, only two studies were conducted in a disguised manner. The first study is multi-centered and was undisguised in the UK but disguised in Germany and France. ${ }^{56}$ The study found prevalence of between 34 and 62 MAEs in every 100 doses observed respectively. However, using the same definition and method, they found 68 MAEs per 100 doses observed undisguised in the UK. The second study that was carried out in a disguised manner was by Feleke and Girma ${ }^{49}$ in Ethiopia. They have found at least 90 MAEs in every 100 doses observed.

Another denominator that was used to represent prevalence of MAEs is the number of opportunities for error. Ghaleb et $\mathrm{al}^{34}$ measured MAEs in ten children's wards using this denominator. They found 19 MAEs in every 100 opportunities for error. This denominator assumes that there are multiple opportunities for error in each dose observed.

Furthermore, a spontaneous self-reporting of MAEs was investigated to collect MAEs that was carried out by Sears et al. ${ }^{40}$ The study was carried out over 3 months in three children's hospitals. A survey tool was developed to capture nurses' self-reporting of MAEs. A total of 372 MAEs were self-reported by registered nurses over 3 months.

\section{Nature of MAEs}

The following are categories reported to be types of MAEs among eight studies in this review. ${ }^{30,33,34,40,41,45,49,56}$ The remaining studies did not report specific MAE subtypes but categorized MAEs along with other ME types:

- Preparation, administration technique, and medicine infusion rate

- Dosage, extra dose, dose omission, and time of administration

- Wrong medicine and unauthorized medicine

- Wrong formulation, wrong route, and wrong strength. In addition, other incidents such as failure to follow hospital rules or policy and administration of doses without double-checking were also classified as types of MAEs.

The most reported therapeutic agent that has been associated with hospital MAEs in children is antimicrobials (between $22.9 \%{ }^{16}$ and $50.3 \%{ }^{49}$ ). Other agents associated with MAEs include anticancer, anticonvulsants, steroids, cardiovascular, opioids, and insulin agents.
Only two studies ${ }^{40,51}$ were found to have measured the severity of MAEs. Chedoe et $\mathrm{al}^{51}$ utilized a panel made up of a doctor, a nurse, and a pharmacist to assess severity independently. Each panelist was asked to rank the potential of harm on a scale of 0 to 10 . This was an interventional study that concluded the pre-intervention harm was $42 \%$ minor $(n=67), 57 \%$ moderate $(n=91)$, and $1 \%$ severe $(n=1)$. Whereas, post-intervention harm was $23 \%$ minor $(n=24)$ and $77 \%$ moderate $(n=80)$. This study carried out an observation of 10 days before and after the intervention. The intervention was based on an educational program, posters for safe preparation and administration, and updated guidelines for the medicine administration process.

The second study that assessed the severity of MAEs was by Sears et al. ${ }^{40}$ The MAE reporter was asked to select the potential of harm. Most of the reports were of minimal error $(n=185)$ and some reported as significant error $(n=112)$. This was followed by serious error $(n=20)$, potentially lethal $(n=51)$, and lethal $(n=4)$.

\section{MAE interventions}

A review of the literature found five types of interventions evaluated to reduce hospital MAEs in children: barcode medicine administration (BCMA), computerized physician order entry (CPOE), education and training, smart pumps, and use of standard concentrations.

\section{BCMA}

This intervention requires patients to wear a barcoded wristband. The barcode contains patient identification details such as patient's name, date of birth, and hospital number that can be integrated with patient's medication record/ prescription. Before each medicine administration, the barcode will need to be scanned against that of the dispensed medicine in order to confirm that the medicine is for the correct patient and is the correct prescribed medicine. This intervention requires streamlining the medication cycle to align with the patient. The dose needs to be prescribed correctly, dispensed, and administered in a standardized approach to the patient. Morriss et $\mathrm{al}^{26}$ observed the number of MEs using a review of medication charts and records kept by the infusion pump. The number of MEs in the pre-intervention phase that did not use BCMA was 39; out of these, 19 were MAEs. In the post-intervention phase of using BCMA, fewer MEs $(n=20)$ were identified. MAEs accounted for 12 incidents. MAEs observed with BCMA related to omission $(n=1)$, wrong dose given $(n=1)$, administration technique $(n=1)$, and wrong time $(n=9)$ errors. 


\section{CPOE}

CPOE is where a prescription is generated electronically for dispensing, and administration. This is also known as electronic prescribing. There have been studies that looked at the use of CPOE and its relation to MAE. The retrospective review by Fontan et $\mathrm{al}^{42}$ found that MAEs are lower with the aid of CPOE (22.5\%) than handwritten prescriptions (29.3\%).

Additionally, Sowan et al ${ }^{24}$ found in a simulation study that CPOE increases the probability of detecting an MAE. The simulation involved 144 infusions that are prepared either against a handwritten prescription or CPOE form. The simulation found that nurses were able to identify $53 \%$ of MAEs in infusions that were ordered using CPOE. On the other hand, nurses identified $40 \%$ of the MAEs in the handwritten infusion prescription. Also, Warrick et $\mathrm{al}^{36}$ evaluated a clinical information system that was integrated with electronic prescribing. This approach has significantly reduced the omitted doses from $8.1 \%$ (43 omitted doses in 528 doses) to $1.4 \%$ (4 omitted doses in 278 doses).

Yamamoto and Kanemori ${ }^{23}$ carried out a prospective comparison between two medication administration practices. The first practice was using a computer-assisted administration that has the prescription integrated and other resources. The second practice was using a conventional method of medicine administration and dosing without access to a computer program or electronic resources. They have found that the frequency of MAEs is lower using computerassisted administration practice ( $\mathrm{n}=27$ MAEs) compared to the conventional method ( $\mathrm{n}=70$ MAEs) with a significant difference $(P<0.001)$. This computer-assisted dosing is a combination of an electronic calculator for preparation and administration of children's medicines.

\section{Education and training}

Educational programs to raise awareness and reduce hospital MAEs in children have been delivered to doctors, nurses, and graduating students. Chedoe et $\mathrm{al}^{51}$ was able to reduce MAEs by $37 \%$ in the Netherlands after implementation of a comprehensive educational program as well as individual nurse training of preparation and administration procedures. The intervention was able to reduce the potential severity of harm. Notably, the wrong administration rate both of minor and moderate harm reduced by $23 \%$ and $12 \%$, respectively. However, the frequency of MAEs such as medication incompatibilities or intravenous lines not flushed increased by $20 \%$ in frequency and severity.

Another educational intervention study by Niemann et a ${ }^{44}$ used a three-step approach of intervention implementation.
They had utilized a comprehensive program of training, informative handouts, and a reference book for medicine administration. The interventions were introduced in a stepwise approach and found that MAEs reduced significantly by $26 \%(P<0.001)$. During the pre-intervention phase, 527 MAEs were identified in 581 drug-handling processes. This was reduced during the post-intervention phase to 116 MAEs in 441 drug-handling processes.

Otero et $\mathrm{al}^{47}$ looked at the effect of comprehensive educational programs for the nursing team. The intervention designed and implemented a "10 steps to reduce medication errors" checklist. The checklist was also provided in a plastic pocket card for nurses to carry around with them. The study pre-intervention MAE rate was $8.4 \%$ and the post-intervention rate reduced to $5.9 \%$ per 100 administered doses. The intervention has reduced dose omission, incorrect dosing, and wrong infusion rate errors.

Another interventional study was done by Bertsche et $\mathrm{al}^{43}$ that aimed to provide training on medicine administration to both the health care team as well as parents. This partnership in improving medication delivery via effective training has significantly reduced hospital MAEs in children. MAE rate pre-intervention was $40.4 \%$ by the health care team and $96.6 \%$ by parents. The post-intervention MAE rate reduced significantly for the nursing team and parents, $7.9 \%$ and $5.6 \%$, respectively, per 100 doses administered.

There have also been interventions that looked at reducing MAEs in the undergraduate education of nursing ${ }^{21,35}$ and medical ${ }^{35}$ students. This is to equip newly graduates with the knowledge and understanding of the medication administration process. Another one is training of staff to improve adherence to hospital regulations and policy by Raja Lope et al. ${ }^{46}$

Moreover, Ellis et a ${ }^{39}$ demonstrated that implementation of new guidelines can prevent harm from MAEs. They have managed to prevent need for the use of morphine antidote or respiratory support in children following administration of a morphine overdose.

A study by Keiffer et $\mathrm{al}^{29}$ investigated the use of educational program in combination of changes to the medication administration process. These changes included: independent double-checking, hands-free communication devices at bedside, ME huddle to identify lessons to be learnt from incidents that caused harm, administration checklist, distraction-free zones by placing a specific floor mat, and use of BCMA. This approach of tackling MAEs was able to reduce the number of events that caused patient harm. Pre-intervention, there were $22 \mathrm{MAE}$ harmful events; this is reduced to three MAE 
events post-intervention. However, MAE is still the most prevalent $\mathrm{ME}$.

\section{Smart pumps and standard concentrations}

Smart pumps are devices with an in-built algorithm that matches the patient's parameters such as weight or body surface area with the correct infusion rate, thereby, decreasing the incidence of MAEs due to the wrong infusion rate, and intercepting prescribing errors due to incorrect calculation of dose and infusion rate.

Trbovich et $\mathrm{al}^{38}$ have evaluated three types of infusion pumps: limited functionality infusion pump, smart infusion pump, and barcode infusion pump. They have found that overall, barcode pumps helped to minimize wrong patient and medicine errors by the nurse scanning a patient wristband against the infusion using a barcode scanner. However, smart infusion pumps were more useful in reducing MAE relating to doses, this is due to the built-in library of medicines that it carries with it. This allows the infusion pump to intercept doses that are outside the safe limits for specific medicines. However, with a limited functionality infusion pump, nurses relied more on their skills and experience. A study by Russell et $\mathrm{al}^{25}$ found that $24 \%$ of medicine observed had a discrepancy between the prescribed dose and the actual dose being given to the patient due to the infusion pumps.

Additionally, Larsen et $\mathrm{al}^{22}$ explored using standard concentrations of medication combined with the use of smart pumps. This combination has resulted in a tenfold decrease of MAEs from 0.41 to 0.08 per 1,000 doses. Another study by Manrique-Rodríguez et $\mathrm{al}^{53}$ that explored the use of smart infusion pumps in the pediatric intensive care unit (PICU) setting found that $78 \%(n=486,875)$ of the infusions were programmed using the in-built library. The ratio of alerts produced by the smart pump was 0.004 . However, no administration error relating to the smart infusion pump was reported. Smart infusion pump intercepted 92 errors; 49\% of these intercepted errors were classified as of moderate, serious, or catastrophic severity.

The use of standardized infusion concentration was also investigated by Bullock et al. ${ }^{27}$ They have found that standardized infusion concentration was able to reduce errors significantly $(P<0.05)$ during the pre-intervention phase: 26 dose errors in 50 infusions, six concentration errors in 26 infusions, and 31 infusions with no standardized concentration in 120 infusions. In comparison, the post-intervention phase had seven dose errors in 28 infusions, zero concentration errors in seven infusions, and 17 infusions with no standardized concentration in 128 infusions.
Another intervention combining use of smart infusion pumps with use of educational materials improved double checking practice, nurse led adverse drug event monitoring,

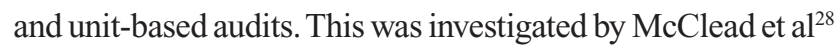
who found that MAEs reduced from $55 \%$ of the total adverse drug events to $38 \%$ over 4 years $(P<0.001)$.

\section{Discussion}

The review was able to explore definitions, methods, and interventions used to investigate MAEs in children's hospitals. Different definitions for MAEs were identified reflecting the diversity in understanding of the problem. However, it was possible to recognize key components that constitute a mutual definition of MAEs. These components consist of an agreement that MAE is a deviation of medicine administered from the prescribed instruction. It also includes errors during the preparation process and the failure to follow hospital standard procedures. Nevertheless, there is a need to develop a definition for MAEs agreed upon by medication safety experts and practitioners that provides a clear and precise statement.

The definition should address issues such as prescribing, dispensing, or monitoring errors that were not intercepted before the administration process. Such errors should also be considered as MAEs since most hospital standard operating procedures for medication administration require staff to conduct adequate clinical checks. The benefit of a standard/agreed MAE definition is that it can reduce heterogeneity of future studies. This will lead to a possibility of meta-analysis and can also be used to conduct randomized control trials of interventions. Also, there is a need for clearer defined subcategories of MAEs. This will enable a better standardization of investigations and interpreting of findings. For example, the majority of the studies identify wrong time, but there is no clear indication of what exactly constitutes the wrong time, and many state it is the administration of the dose \pm 1 hour of scheduled time. Others state it is \pm 30 minutes. The impact of these differences can be vital since it will affect the number of MAEs identified. Therefore, this is a major cause for heterogeneity of the data.

Furthermore, this review found various methods used to investigate hospital MAEs in children. There are indeed strengths and limitations for each method but a triangulation of methodological approach to study hospital MAEs in children will lead to a better understanding of the true nature, causes, and severity of the problem.

There were variations in the denominators of which MAEs were expressed. This could cause confusion, misunderstanding, or irregularity in interpreting MAE prevalence. Also, the number of MAEs detected prospectively is dependent on factors like observer clinical experience and knowledge of 
the medicine administration process. Similar concerns were expressed in the review by McLeod et al. ${ }^{8}$

The review found heterogeneity between studies due to: study setting, definitions, size, duration of study, and tools used to identify hospital MAEs in children. If this heterogeneity is to be neglected, the review would find cumulatively a total of 12,588 ME reported incidents in children's hospitals; of these, MAEs account for $50 \%(n=6,247)$. Whereas, using a prospective observation method, a total of 2,537 MAEs (29\%) were detected when 8,894 doses were observed. These findings demonstrate the scale of the problem when providing medicine to children in hospital. Yet, data are limited regarding the level of harm this causes or the potential for harm.

Antimicrobials agents were found to be the medicine most commonly associated with MAEs. This finding is expected since antimicrobials are considered the most prescribed agents in this cohort. However, this is also due to difficulties in dose calculations, giving it at the correct time intervals, or the preparation of intravenous infusions. Moreover, this could have been as a result of other errors not intercepted in the prescribing or dispensing process. Especially as many medications are used in an unlicensed and/or off-label manner in children. The review found only one study that carried out an assessment of the potential harm of MAEs. Another gap in the literature is that no study has carried out exploring the contributory factors of MAEs in PICU. This is important since both knowledge of severity of harm and contributing factors can help to develop interventions and will facilitate the development of interventions that focus on risky practices by both the system and practitioners.

In addition, interventional studies in hospital MAEs were explored and categorized as: BCMA, electronic prescribing, education and training, use of smart pumps, and standard concentration. However, evidence is not strong enough to support their true impact or effects on reducing MAEs. This is possibly due to the multifaceted nature of MAEs.

For example, BCMA has shown the advantages of reducing MAEs relating to wrong patient, wrong medicine, and time errors. However, other MAEs may not be reduced such as errors in preparation, administration technique, or wrong dose. Additionally, implementation of this BCMA system will encounter financial costs and require staff training to ensure competence.

On the other hand, CPOE allows clearer dosage instructions than handwritten prescriptions, hence minimizing the risk of giving the wrong dose, and can help detect prescribing errors before administering the medicine. Also, use of pharmaceutical calculation aids can provide better personalized clinical decisions. Therefore, this will help in ensuring correct administration of medicines by providing information of the amount of drug to draw out, the diluent volume, and the infusion rate. However, this approach may not address issues such as wrong patient, wrong time, or wrong medicine and may introduce new errors, such as discrepancies between what is electronically recorded and what is actually given. Therefore, more evaluation is required of these interventions and a study of the impact of multiple interventions on MAEs.

This review suggests that there is a need for more than one intervention to reduce MAEs in practice. They should focus on supporting good medication safety practices that include no blame culture, promote learning from errors, and involve new technologies. Nevertheless, it is equally important to put in place suitable monitoring methods over long periods of time to assess the suitability of interventions.

However, the review identified a key gap in literature and that is the limited number of interventions in PICUs. This is despite the PICU being a high-risk area that provides round the clock medicine administration. Many of these medicines are of narrow therapeutic window and use of unlicensed/ off-label medicines are frequent. Although, there have been a number of studies carried out in PICU to quantify the scale of the problem. Additionally, no study was found which investigated the opinions of PICU health care professionals into the causes of MAEs or sought recommendations from them to improve their administration practice. As far as the researchers are aware, there were only two studies carried out in the UK that tried to reduce MAEs. ${ }^{35,36}$ Both studies did not actually address MAEs fully nor did they reduce MAEs in PICUs. Stewart et $\mathrm{al}^{35}$ investigated the use of education and simulation of the administration process with undergraduate nurse students. The study is of less application in real practice since the study was carried out over a very short period of time. Also, they did not follow-up the students' performance in real time clinical situations. On the other hand, Warrick et $\mathrm{al}^{36}$ utilized a clinical information system for providing support for doctors and nurses. However, they only measured MAEs in terms of omitted doses. This is by no means a reflection of the true level of the problem in practice. Also, they carried out the study in an emergency department where patients are only likely to stay for a very short period of time. Hence, many of the scheduled doses will be missed because they would have either been discharged to another ward or sent back home. Therefore, there is a serious need for evidence-based MAE safety measures in PICU.

This review builds upon knowledge found by other researchers. ${ }^{6,8,9,11}$ It also agrees with Raban and Westbrook ${ }^{13}$ that reducing interruption alone is associated with a weak evidence of reducing MAEs. 
This review did not identify research written in nonEnglish language due to time constraints and lack of translators. Inclusion of such research would add vital insight into the type of research and nature of MAEs in different parts of the world. However, the databases that were searched did return a number of non-English studies that were judged not to be relevant for this review. A separate search on non-English databases would be required along with a trusted translator who has experience in conducting literature reviews.

Additionally, only interventional studies related to hospital MAEs were reviewed, but it would have been beneficial to review overall ME interventions as well since this review has shed light on the multifaceted nature of MAEs. Also, this review has focused only on hospital interventions to MAEs in children. A separate review is recommended specifically to identify all the MAE interventions in both adults and children for allowing shared learning and adaptation of interventions across different settings.

Future MAE research should address the need for a validated expert consensus on a clear practical guide to carry out MAE studies along with a standard approach to define, classify, and report MAEs. This will result in a better understanding of the problem and lead to development of evidence-based interventions.

\section{Conclusion}

This review has identified wide variation in the prevalence of hospital MAEs in children. This is attributed to the methods and definitions used to investigate these errors as identified in this review. Additionally, the review found weak evidence for a single intervention in reducing hospital MAEs in children. This illustrates the complexity and multifaceted nature of this issue. Therefore, there is a need to develop a set of safety measures to tackle these errors.

\section{Acknowledgment}

AA would like to thank the Department of Pharmacy, University of Hertfordshire, for sponsoring his $\mathrm{PhD}$ research program.

\section{Disclosure}

The authors report no conflicts of interest in this work.

\section{References}

1. Kaushal R, Bates DW, Landrigan C, et al. Medication errors and adverse drug events in pediatric inpatients. JAMA. 2001;285(16):2114-2120.

2. Kohn LT, Corrigan JM, Donaldson MS, editors. To Err Is Human: Building a Safer Health System. Washington, DC: National Academies Press; 2000. Available from: http://www.nap.edu/openbook.php?record_ id=9728. Accessed March 15, 2012.
3. Gonzales K. Medication administration errors and the pediatric population: a systematic search of the literature. J Pediatr Nurs. 2010; 25(6):555-565.

4. Giacoia GP, Taylor-Zapata P, Mattison D. Need for appropriate formulations for children: The National Institute of Child Health and Human Development - Pediatric Formulations Initiative, Part 1. Int J Pharm Compd. 2007;11(1):5-8.

5. Conroy S, Appleby K, Rostock D, Unsworth V, Cousins D. Medication errors in a children's hospital. Paediatr Perinat Drug Ther. 2007;8(1): $18-25$.

6. Ghaleb MA, Barber N, Franklin BD, Yeung VW, Khaki ZF, Wong IC. Systematic review of medication errors in pediatric patients. Ann Pharmacother. 2006;40(10):1766-1776.

7. Conroy S. Association between licence status and medication errors. Arch Dis Child. 2011;96(3):305-306.

8. McLeod MC, Barber N, Franklin BD. Methodological variations and their effects on reported medication administration error rates. $B M J$ Qual Saf. 2013;22(4):278-289.

9. Keers RN, Williams SD, Cooke J, Ashcroft DM. Prevalence and nature of medication administration errors in health care settings: a systematic review of direct observational evidence. Ann Pharmacother. 2013;47(2):237-256

10. Miller MR, Robinson KA, Lubomski LH, Rinke ML, Pronovost PJ. Medication errors in paediatric care: a systematic review of epidemiology and an evaluation of evidence supporting reduction strategy recommendations. Qual Saf Health Care. 2007;16(2):116-126.

11. Rinke ML, Bundy DG, Velasquez CA, et al. Interventions to reduce pediatric medication errors: a systematic review. Pediatrics. 2014;134(2):338-360.

12. Keers RN, Williams SD, Cooke J, Ashcroft DM. Causes of medication administration errors in hospitals: a systematic review of quantitative and qualitative evidence. Drug Saf. 2013;36(11):1045-1067.

13. Raban MZ, Westbrook JI. Are interventions to reduce interruptions and errors during medication administration effective? A systematic review. BMJ Qual Saf. 2013;23:414-421.

14. PRISMA. The PRISMA statement. 2014. Available from: http://www. prisma-statement.org/statement.htm. Accessed August 15, 2014.

15. Hicks RW, Becker SC, Windle PE, Krenzischek DA. Medication errors in the PACU. J Perianesth Nurs. 2007;22(6):413-419.

16. Miller AD, Piro CC, Rudisill CN, Bookstaver PB, Bair JD, Bennett CL. Nighttime and weekend medication error rates in an inpatient pediatric population. Ann Pharmacother. 2010;44(11):1739-1746.

17. Stavroudis TA, Shore AD, Morlock L, Hicks RW, Bundy D, Miller MR. NICU medication errors: identifying a risk profile for medication errors in the neonatal intensive care unit. J Perinatol. 2010;30(7):459-468.

18. Suresh G, Horbar JD, Plsek P, et al. Voluntary anonymous reporting of medical errors for neonatal intensive care. Pediatrics. 2004;113(6): 1609-1618.

19. Herout PM, Erstad BL. Medication errors involving continuously infused medications in a surgical intensive care unit. Crit Care Med. 2004;32(2):428-432.

20. Marino BL, Reinhardt K, Eichelberger WJ, Steingard R. Prevalence of errors in a pediatric hospital medication system: implications for error proofing. Outcomes Manag Nurs Pract. 2000;4(3):129-135.

21. Pauly-O'Neill S. Beyond the five rights: improving patient safety in pediatric medication administration through simulation. Clin Sim Nur. 2009;5(5):s181-s186.

22. Larsen GY, Parker HB, Cash J, O'Connell M, Grant MJC. Standard drug concentrations and smart-pump technology reduce continuousmedication-infusion errors in pediatric patients. Pediatrics. 2005;116(1): E21-E25.

23. Yamamoto L, Kanemori J. Comparing errors in ED computer-assisted vs conventional pediatric drug dosing and administration. Am J Emerg Med. 2010;28(5):588-592.

24. Sowan AK, Gaffoor MI, Soeken K, Johantgen ME, Vaidya VU. Impact of computerized orders for pediatric continuous drug infusions on detecting infusion pump programming errors: a simulated study. J Pediatr Nurs. 2010;25(2):108-118. 
25. Russell RA, Murkowski K, Scanlon MC. Discrepancies between medication orders and infusion pump programming in a paediatric intensive care unit. Qual Saf Health Care. 2010;19:i31-i35.

26. Morriss FH Jr, Abramowitz PW, Nelson SP, et al. Effectiveness of a barcode medication administration system in reducing preventable adverse drug events in a neonatal intensive care unit: a prospective cohort study. J Pediatr. 2009;154(3):363-368.

27. Bullock J, Jordan D, Gawlinski A, Henneman EA. Standardizing IV infusion medication concentrations to reduce variability in medication errors. Crit Care Nurs Clin North Am. 2006;18(4):515-521.

28. McClead RE Jr, Catt C, Davis JT, et al. An internal quality improvement collaborative significantly reduces hospital-wide medication error related adverse drug events. J Pediatr. 2014;165(6):1222-1229. e1.

29. Keiffer S, Marcum G, Harrison S, Teske DW, Simsic JM. Reduction of medication errors in a pediatric cardiothoracic intensive care unit. J Nurs Care Qual. 2015;30(3):212-219.

30. Ross LM, Paton JY, Wallace J. Medication errors in a paediatric teaching hospital in the UK: five years operational experience. Arch Dis Child. 2000;83(6):492-497.

31. Simpson JH, Lynch R, Grant J, Alroomi L. Reducing medication errors in the neonatal intensive care unit. Arch Dis Child. 2004;89(6): F480-F482.

32. Thomas AN, Panchagnula U. Medication-related patient safety incidents in critical care: A review of reports to the UK National Patient Safety Agency. Anaesthesia. 2008;63(7):726-733.

33. Alsulami Z, Choonara I, Conroy S. Paediatric nurses' adherence to the double-checking process during medication administration in a children's hospital: an observational study. J Adv Nurs. 2014;70(6): 1404-1413.

34. Ghaleb MA, Barber N, Franklin BD, Wong IC. The incidence and nature of prescribing and medication administration errors in paediatric inpatients. Arch Dis Child. 2010;95(2):113-118.

35. Stewart M, Purdy J, Kennedy N, Burns A. An interprofessional approach to improving paediatric medication safety. BMC Med Educ. 2010;10:19.

36. Warrick C, Naik H, Avis S, Fletcher P, Franklin BD, Inwald D. A clinical information system reduces medication errors in paediatric intensive care. Intensive Care Med. 2011;37(4):691-694.

37. Doherty C, McDonnell C. Tenfold medication errors: 5 years' experience at a university-affiliated pediatric hospital. Pediatrics. 2012 129(5):916-924.

38. Trbovich PL, Pinkney S, Cafazzo JA, Easty AC. The impact of traditional and smart pump infusion technology on nurse medication administration performance in a simulated inpatient unit. Qual Saf Health Care. 2010;19(5):430-434.

39. Ellis J, Martelli B, Lamontagne C, et al. Improved practices for safe administration of intravenous bolus morphine in a pediatric setting. Pain Manag Nurs. 2011;12(3):146-153.

40. Sears K, O’Brien-Pallas L, Stevens B, Murphy GT. The relationship between the nursing work environment and the occurrence of reported paediatric medication administration errors: a pan Canadian study. J Pediatr Nurs. 2013;28(4):351-356.

41. Prot S, Fontan JE, Alberti C, et al. Drug administration errors and their determinants in pediatric in-patients. Int J Qual Health Care. 2005 17(5):381-389.
42. Fontan J, Maneglier V, Nguyen V, Loirat C, Brion F. Medication errors in hospitals: computerized unit dose drug dispensing system versus ward stock distribution system. Pharm World Sci. 2003;25(3):112-117.

43. Bertsche T, Bertsche A, Krieg E, et al. Prospective pilot intervention study to prevent medication errors in drugs administered to children by mouth or gastric tube: a programme for nurses, physicians and parents. Qual Saf Health Care. 2010;19(5):e26.

44. Niemann D, Bertsche A, Meyrath D, et al. A prospective three-step intervention study to prevent medication errors in drug handling in paediatric care. J Clin Nurs. 2015;24(1-2):101-114.

45. Chua SS, Chua HM, Omar A. Drug administration errors in paediatric wards: a direct observation approach. Eur J Pediatr. 2010;169(5): 603-611.

46. Raja Lope RJ, Boo NY, Rohana J, Cheah FC. A quality assurance study on the administration of medication by nurses in a neonatal intensive care unit. Singapore Med J. 2009;50(1):68-72.

47. Otero P, Leyton A, Mariani G, Ceriani Cernadas JM; Patient Safety Committee. Medication errors in pediatric inpatients: prevalence and results of a prevention program. Pediatrics. 2008;122(3):e737-e743.

48. Manias E, Kinney S, Cranswick N, Williams A. Medication errors in hospitalised children. J Paediatr Child Health. 2014;50(1):71-77.

49. Feleke Y, Girma B. Medication administration errors involving paediatric in-patients in a hospital in Ethiopia. Trop J Pharm Res. 2010;9(4): 401-407.

50. Sakuma M, Ida H, Nakamura T, et al. Adverse drug events and medication errors in Japanese paediatric inpatients: a retrospective cohort study. BMJ Qual Saf. 2014;23(10):830-837.

51. Chedoe I, Molendijk H, Hospes W, Van den Heuvel ER, Taxis K. The effect of a multifaceted educational intervention on medication preparation and administration errors in neonatal intensive care. Arch Dis Child Fetal Neonatal Ed. 2012;97(6):F449-F455.

52. Sadat-Ali M, Al-Shafei BA, Al-Turki RA, Ahmed SE, Al-Abbas SA, Al-Omran AS. Medication administration errors in Eastern Saudi Arabia. Saudi Med J. 2010;31(11):1257-1259.

53. Manrique-Rodríguez S, Sánchez-Galindo AC, López-Herce J, et al. Impact of implementing smart infusion pumps in a pediatric intensive care unit. Am J Health Syst Pharm. 2013;70(21):1897-1906.

54. Frey B, Buettiker V, Hug MI, et al. Does critical incident reporting contribute to medication error prevention? Eur J Pediatr. 2002;161(11) 594-599.

55. Ozkan S, Kocaman G, Ozturk C, Seren S. Frequency of pediatric medication administration errors and contributing factors. J Nurs Care Qual. 2011;26(2):136-143.

56. Cousins DH, Sabatier B, Begue D, Schmitt C, Hoppe-Tichy T. Medication errors in intravenous drug preparation and administration: a multicentre audit in the UK, Germany and France. Qual Saf Health Care. 2005;14(3):190-195.

57. Conroy S, Sweis D, Planner C, Yeung V, Collier J, Haines L, Wong IC. Interventions to reduce dosing errors in children: a systematic review of the literature. Drug Saf. 2007;30(12):1111-1125.

58. Stavroudis TA, Miller MR, Lehmann CU. Medication errors in neonates. Clin Perinatol. 2008;35(1):141-161.
Integrated Pharmacy Research and Practice

\section{Publish your work in this journal}

Integrated Pharmacy Research and Practice is an international, peer-reviewed, open access, online journal, publishing original research, reports, reviews and commentaries on all areas of academic and professional pharmacy practice. This journal aims to represent the academic output of pharmacists and pharmacy practice with particular focus on integrated care. All papers are carefully

\section{Dovepress}

peer reviewed to ensure the highest standards as well as ensuring that we are informing and stimulating pharmaceutical professionals. The manuscript management system is completely online and includes a very quick and fair peer-review system, which is all easy to use. Visit http://www.dovepress.com/ testimonials.php to read real quotes from published authors. 